\title{
Delayed Galaxies
}

\section{Curtis Struck ${ }^{1}$, Mark Hancock ${ }^{2}$, Beverly J. Smith ${ }^{2}$, Phillip N. Appleton $^{3}$, Vassilis Charmandaris ${ }^{4}$ and Mark Giroux ${ }^{2}$}

\author{
${ }^{1}$ Department of Physics and Astronomy, Iowa State University, Ames IA 50011, \\ email: curt@iastate.edu \\ ${ }^{2}$ Department of Physics, Astronomy, and Geology, East Tennessee State University, Johnson \\ City TN 37614 \\ ${ }^{3}$ Spitzer Science Center, California Institute of Technology, Pasadena CA 91125 \\ ${ }^{4}$ Department of Physics, University of Crete, Heraklion Greece 71003
}

\begin{abstract}
We can define Delayed Galaxies as a class of rare galaxies that maintained the bulk of their gas for most of the age of the universe following the initial formation of their disks, with little or no star formation. Invisible galaxies and Malin 1 type low-surface-brightness galaxies qualify as class members. Rare examples among interacting galaxies show that collisions can restart the stalled evolution of such galaxies, and suggest that other members of the Delayed class can be found among interacting systems with vigorous current star formation.
\end{abstract}

Keywords. galaxies: interacting, galaxies: evolution, galaxies: formation

The subject of this paper is a class of galaxies that were recently, but are not presently, invisible... or nearly invisible. Currently, they are quite spectacularly visible, thanks to substantial rates of star formation. That is, samples of interacting galaxies contain rare ones with no old stellar population in evidence. I would quickly add the caveat that the observational limits are such that the phrase "no old population" is probably too strong. The statement "dominant young-to-intermediate populations" is more accurate at this stage. The other caveat that should be given at the outset is that too few of these objects are known to make any reliable statements about the class characteristics. I will focus on the example of the Arp 82 system, mention the case of the Cartwheel ring galaxy, and here note that if VIRGO21HI is an invisible galaxy, it may soon join this group as a result of its most recent interaction, or other interactions in its future.

There is another sub-group on the edge of the class of delayed, interacting galaxies. These are the companions in M51-type encounters (prograde interactions as viewed from the primary, and slightly bound), which are significantly smaller than the primary galaxy, but still massive enough to significantly perturb the primary. Roughly, the relevant mass ratio of primary-to-companion ranges from a few to ten. In such cases a large amount of gas can be transferred via a tidal bridge from the outer disk of the primary to the companion (e.g., Toomre \& Toomre 1972, Wallin \& Stuart 1992). If this gas mass is of the order already possessed by the companion, it can contribute substantially to the evolution of the companion, just as though it had kept that gas in its own disk reservoir for many Gyr. Yet this is really a different kind of delay, and probably not terribly important, since such companions generally merge quite promptly, so we will not consider this case any further.

Instead, let us move on to a little background information on our primary example, the Arp 82 system (see Figure 1). The primary galaxy (NGC 2535) in this system has a long tidal tail and strong bridge connecting to the companion (NGC 2536). It also has an ocular shaped ring within its disk. All of these features are indicative of a prolonged prograde interaction of this galaxy with its companion. Though relatively nearby (distance 

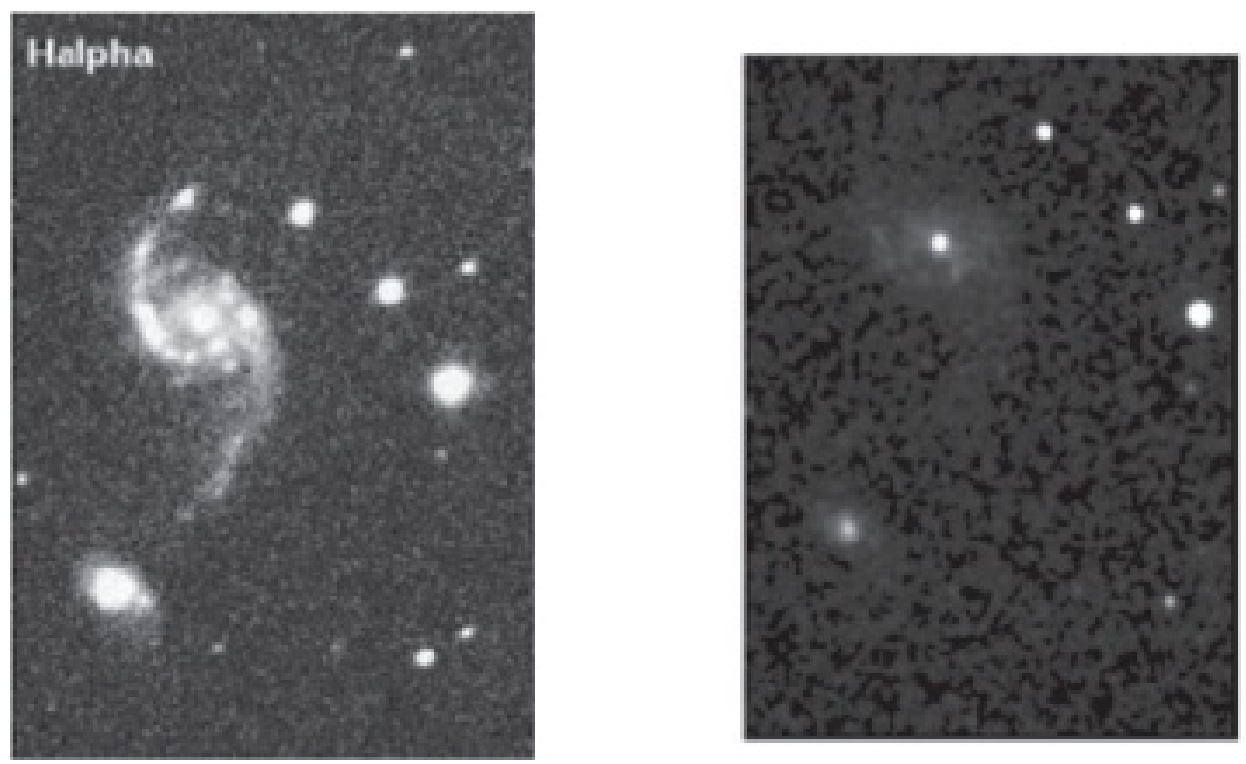

Figure 1. The Arp 82 system shown in an $\mathrm{H} \alpha$ image from Hancock et al. (2007a) on the left, and a 2MASS Ks image on the right. The Ks image also conveys a fair impression of what the system might have looked like in the optical during its long quiescent (delayed) history.

$59.8 \mathrm{Mpc}$, recession velocity $4097 \mathrm{~km} \mathrm{~s}^{-1}$, according to NED), the system had not been much studied before the 1990s. However, it was noted as a prime example of the ocular class by Elmegreen et al. (1991). Amran et al. (1989) subsequently used Fabry-Perot observations to study its optical velocity field, and Kaufman et al. (1997) presented VLA mapping observations of its $\mathrm{HI}$ gas.

More recently, this system was included in the sample of the Spirals, Bridges and Tails (SB\&T) project, for which our collaboration obtained Spitzer Space Telescope, GALEX, and ground-based observations of a few dozen interacting galaxy systems (Smith et al. 2007). It was one of the first few systems from that sample for which we have undertaken a multi-waveband analysis of the star-forming clumps (Hancock et al. 2007a). Specifically, we have fitted Starburst99 models to the optical and GALEX UV data, and found moderate extinctions and young ages for the clump sources. The median age of the clumps was found to be about $9 \mathrm{Myr}$. This is an interesting result in itself, since numerical models suggest an interaction timescale of hundreds of Myr, so this system evidently provides another example of rapid infant mortality of star clusters in interacting systems (e.g., Lamers 2007, Scheepmaker 2007, Whitmore, Chandar \& Fall 2007).

What is even more interesting is that analysis of the colors of the diffuse, non-clump, emission revealed young-to-intermediate ages. In particular, there is no evidence for a stellar population much older than about 2 Gyr. I.e., the remaining stellar emission might originate only in stars formed since the onset of the interaction (though if the timescale is really as long as $2 \mathrm{Gyr}$, this might include a close passage before the most recent one). It may be that the diffuse emission is entirely due to stars from clusters formed and subsequently disrupted via infant mortality processes.

At present this result should be viewed cautiously. This system has inspired us to undertake an extensive grid of Starburst99 models, to compare to well-studied Galactic star clusters, with the goal of accessing the uncertainties in ages derived as above (Hancock 
et al. 2007b). We are currently working to derive specific age uncertainties for the clumps and diffuse regions of Arp 82.

We note that there is corroborating evidence for the conjecture that Arp 82 is a long delayed system. The system is unusually gas rich, with an HI mass comparable to the mass of stars (of order a few times $10^{10} M_{\odot}$, Kaufman et al. 1997), which in itself suggests that it is not highly evolved. Overall the galaxies are of intermediate mass, so the system also seems to be an example of downsizing in the present day.

On the other hand, there are also several pieces of evidence to suggest that this is not a truly young galaxy in the throes of formation. First of all, there are the tidal structures themselves, which are characteristic of a perturbed disk. That is, the formation of such structures requires a pre-existent disk, even if that disk was not able to form many stars. The HI kinematics are indicative of a perturbed disk, not an ill-formed one (Kaufman et al. 1997). The kinematic range also indicates the presence of a dark matter halo (Kaufman et al. 1997, Amram et al. 1989). In general, a significant metallicity value would also support this conclusion, but metallicity information is not yet available for Arp 82.

Finally, we note that this system seems to be isolated, with no nearby galaxies visible on optical images. In general, we would expect delayed galaxies to be preserved in very low density environments.

The well-known Cartwheel ring galaxy may be another galaxy in the class of delayed until triggered by the current interaction. However, it is apparently not such an extreme example. There are old stars in its central regions, and evidence that there was an old stellar disk, of much smaller size than the present ring (Vorobyov \& Bizyaev 2001). It has a near-solar metallicity in its inner regions (Vorobyov \& Bizyaev 2001), and a high abundance of heated dust (Charmandaris et al. 1999). On the other hand, it has about $10^{10} M_{\odot}$ of HI within its disk (and apparently more splashed out, Higdon 1996), and (1.5 6.0) $\times 10^{9} M_{\odot}$ of molecular gas in its central regions (Horelou, et al. 1998). The mass of old stars in the central regions is probably more than an order of magnitude lower than the total gas mass (Vorobyov \& Bizyaev 2001), so its progenitor was either of very late type or an LSB galaxy. In fact, the progenitor may have looked much like Malin 1, which also has a small, ordinary galaxy in the center of its huge HI disk (Pickering et al. 1997, Moore \& Parker 2006, Barth 2007).

Giant low surface brightness galaxies (LSBs) are very rare (see paper of K. ONeill at this meeting), and strongly interacting galaxies are also rare in the world of galaxies. However, the class of strongly interacting galaxies with LSB progenitors might not be as rare as the product of the relative abundances of the two classes. Neither class is commonly found in dense environments; the extended gas disks of LSBs would be quickly stripped in a large cluster environment, and high relative velocity collisions, which yield weak interactions, are more common there. LSBs and strongly interacting galaxies are more likely to be found in small groups. The implication is that LSBs are more likely to be progenitors of strong interactions than if progenitors were chosen randomly from all galaxies. Thus, Arp 82 and the Cartwheel may not be as unusual as it seems at first sight.

At present, these points cannot be quantified because we have so few examples. However, if they are significant, they have another interesting ramification in estimating the SF enhancements in interactions we may have been comparing to a biased control sample of isolated galaxies. For example, in analyzing the SB\&T sample we used SINGS galaxies as a control sample, in part, because there was no other comparably large sample of normal galaxies to compare to. Even so, we had to reject a few strongly interacting systems included in SINGS. Yet, in the end, of the 26 galaxies in our spiral control sample 4 
are Virgo cluster galaxies (including NGC 4254, the associate of VIRGOHI21), and thus, drawn from a very different environment than most of our interacting systems (see Smith et al. 2007). More of the spiral control objects are Messier objects, and thus, inevitably of high surface brightness. This spiral control sample also still includes a few weakly interacting systems, like NGC 4254 and NGC 1097.

This bias may be particularly bad in wavebands with few available isolated galaxy samples, like the mid-IR. It might be less of a problem in large optical samples, with large control samples, e.g., in recent work with the SDSS and $2 \mathrm{dF}$ samples (e.g., Owers et al. 2007, Woods \& Geller 2007, and references therein), but in the optical band one faces problems with extinction corrections. In no published study that I am aware of, has an explicit effort been made to draw control objects from the same types of environment as interacting galaxies, so we may yet not have a good handle on how SF is enhanced in interactions at early stages.

In sum, although there are currently many unknowns, there are reasons to think that a good place to search for delayed, recently invisible galaxies is among the newly lit-up galaxies in strongly interacting systems.

\section{Acknowledgements}

We thank the Spitzer, GALEX, and SDSS teams for making this research possible. This research was supported by NASA Spitzer grants 1263924 and 1301516, and NASA GALEX grant GALEXGI04-0000-0026. This research has made use of the NASA/IPAC Extragalactic Database (NED) which is operated by the Jet Propulsion Laboratory, California Institute of Technology, under contract with the National Aeronautics and Space Administration.

\section{References}

Amran, P., Marcelin, M., Boulesteix, J., \& le Coarer, E. 1989, A\&AS 81, 59

Barth, A. J. 2007, $A J, 133,1085$

Charmandaris, V., et al. 1999, A\&A, 341, 69

Elmegreen, D. M., Sundin, M., Elmegreen, B. G., \& Sundelius, B. 1991 A\& A, 244, 52

Hancock, M. et al. 2007a, AJ, 133, 676

Hancock, M. et al. 2007b, $A J$, submitted

Higdon, J. L. 1996, ApJ, 467, 241

Horellou, C., Charmandaris, V., Combes, F., Appleton, P. N., Casoli, F., \& Mirabel, I. F. 1998 $A \& A, 340, \mathrm{~L} 51$

Kaufman, M., et al. 1997, AJ, 114, 2323

Lamers, H. J. G. L. M. 1997, arXiv:0704.3159

Moore, L., \& Parker, Q. A. 2006, PASA, 23, 165

Owers, M. S., Blake, C., Couch, W. J., Pracy, M. B., \& Bekki, K. 2007, MNRAS, submitted (arXiv:0707.2566)

Pickering T. E., Impey, C. D., van Gorkom, J. H., \& Bothun, G. D. 1997, AJ, 114, 1858

Scheepmaker, R. A., et al. 2007, AJ, 114, 2323

Smith, B. J., Struck, C., Hancock, M., Appleton, P. N., Charmandaris, V., \& Reach, W. 2007, AJ, 133, 791

Toomre, A., \& Toomre, J. 1972, ApJ, 178, 623

Vorobyov, E. I., \& Bizyaev, D. 2001, A\& A, 377, 835

Wallin, J. F., \& Stuart, B. V. 1992, ApJ, 339, 29

Whitmore, B. C., Chandar, R., \& Fall, S. M. 2007, AJ, 133, 1067

Woods, D. F., \& Geller, M. J. 2007, AJ, 134, 527 